\title{
A General Solution for Troesch's Problem
}

\author{
Hector Vazquez-Leal, ${ }^{1}$ Yasir Khan, ${ }^{2}$ \\ Guillermo Fernández-Anaya, ${ }^{3}$ Agustín Herrera-May, ${ }^{4}$ \\ Arturo Sarmiento-Reyes, ${ }^{5}$ Uriel Filobello-Nino, ${ }^{1}$ \\ Víctor-M. Jimenez-Fernández, ${ }^{1}$ and Domitilo Pereyra-Díaz ${ }^{1}$ \\ ${ }^{1}$ Electronic Instrumentation and Atmospheric Sciences School, University of Veracruz, \\ Xalapa, VER 91000, Mexico \\ ${ }^{2}$ Department of Mathematics, Zhejiang University, Hangzhou 310027, China \\ ${ }^{3}$ Departamento de Física y Matemáticas, Universidad Iberoamericana, Prolongación Paseo de \\ la Reforma 880, 01219 Mexico DF, Mexico \\ ${ }^{4}$ Micro and Nanotechnology Research Center, University of Veracruz, Calzada Ruiz Cortines 455, \\ 94292 Boca del Rio, VER, Mexico \\ ${ }^{5}$ National Institute for Astrophysics, Optics and Electronics Luis Enrique Erro No.1, \\ 72840 Santa María Tonantzintla, PUE, Mexico
}

Correspondence should be addressed to Hector Vazquez-Leal, hvazquez@uv.mx

Received 23 August 2012; Accepted 6 October 2012

Academic Editor: Farzad Khani

Copyright (C) 2012 Hector Vazquez-Leal et al. This is an open access article distributed under the Creative Commons Attribution License, which permits unrestricted use, distribution, and reproduction in any medium, provided the original work is properly cited.

The homotopy perturbation method (HPM) is employed to obtain an approximate solution for the nonlinear differential equation which describes Troesch's problem. In contrast to other reported solutions obtained by using variational iteration method, decomposition method approximation, homotopy analysis method, Laplace transform decomposition method, and HPM method, the proposed solution shows the highest degree of accuracy in the results for a remarkable wide range of values of Troesch's parameter.

\section{Introduction}

Troesch's equation is a boundary value problem (BVP) expressed as

$$
y^{\prime \prime}=n \sinh (n y), \quad y(0)=0, y(1)=1,
$$

where prime denotes differentiation with respect to $x$ and $n$ is known as Troesch's parameter. 
Equation (1.1) arises in the investigation of confinement of a plasma column by a radiation pressure [1] and also in the theory of gas porous electrodes [2,3]. This BVP problem has a pole [4] approximately located at

$$
x_{\infty}=\frac{1}{n} \ln \left(\frac{8}{y^{\prime}(0)}\right)
$$

which makes the solution of (1.1) a difficult task for numerical methods.

In order to overcome such difficulties, there are several reported numerical solutions for Troesch's problem [4-10]. Recently, after some decades using wrong numerical results, in $[8,9]$ were reported the most accurate solutions for $n=0.5$ and $n=1$. Besides, there are approximated analytical solutions obtained by using different methods like homotopy perturbation method (HPM) [11, 12], decomposition method approximation (DMA) [12, 13], homotopy analysis method (HAM) [14], variational iteration method (VIM) [15], and Laplace transform decomposition method (LTDM) [16]. The main disadvantage of aforementioned approximated solutions is that they are obtained for specific values of $n$, like $n=0.5,1$ or 10 . In contrast, we propose a general approximate solution for Troesch's problem, useful for $n \geq 0$, by using HPM method [17-27].

This paper is organized as follows. In Section 2, we provide a brief review of HPM method. In Section 3, we obtain the solution of Troesch's problem employing HPM. Section 4 shows numerical simulations and discuss our findings. Finally, a brief conclusion is given in Section 5.

\section{Basic Idea of HPM Method}

Basically, the HPM method [17-26, 31-53] introduces a homotopy parameter $p$, which takes values ranging from 0 up to 1 . When parameter $p=0$, the equation usually reduces to a simple, or trivial, equation to solve. Then, $p$ is gradually increased to 1 , producing a sequence of deformations. Eventually, at $p=1$, the homotopy equation takes the original form of the equation to solve, and the final stage of deformation provides the desired solution. Usually, few iterations are required to obtain good results [17-19].

In the HPM method, it is considered that a nonlinear differential equation can be expressed as

$$
A(u)-f(r)=0, \quad r \in \Omega,
$$

with the boundary condition

$$
B\left(u, \frac{\partial u}{\partial \eta}\right)=0, \quad r \in \Gamma
$$

where $A$ is a general differential operator, $f(r)$ is a known analytic function, $B$ is a linear initial/boundary operator, $\Gamma$ is the boundary of domain $\Omega$, and $\partial u / \partial \eta$ denotes differentiation along the normal drawn outwards from $\Omega$. The $A$ operator, generally, can be divided into two 
operators, $L$ and $N$, where $L$ is the linear operator, and $N$ is the nonlinear operator. Hence, (2.1) can be rewritten as

$$
L(u)+N(u)-f(r)=0 .
$$

Now, a possible homotopy formulation is

$$
H(v, p)=(1-p)\left[L(v)-L\left(u_{0}\right)\right]+p(L(v)+N(v)-f(r))=0, \quad p \in[0,1]
$$

where $u_{0}$ is the initial approximation for the solution of (2.3) which satisfies the boundary conditions, and $p$ is known as the perturbation homotopy parameter.

We assume that the solution of (2.4) can be written as a power series of $p$ as

$$
v=p^{0} v_{0}+p^{1} v_{1}+p^{2} v_{2}+\cdots
$$

When $p \rightarrow 1$, results that the approximate solution for (2.1) is

$$
u=\lim _{p \rightarrow 1} v=v_{0}+v_{1}+v_{2}+\cdots
$$

The series (2.6) is convergent on most cases, nevertheless, the convergence depends on the nonlinear operator $A(v)[19,21,35,36,52]$.

\section{Solution of Troesch's Problem by Using HPM Method}

Straight forward application of HPM to solve (1.1) is not possible due to the hyperbolic sin term of dependent variable. However, in [11, 12] were reported HPM solutions, obtained by using a power series expansion of the sinh term of (1.1), which are limited to $n \leq 1$, due to the truncate power series. Nevertheless, the polynomial type nonlinearities are easier to handle by the HPM method. Therefore, in order to apply HPM successfully for a wide range of Troesch's parameter $(n)$, we convert the hyperbolic-type nonlinearity in Troesch's problem into a polynomial type nonlinearity, using the variable transformation reported in [15]. First, we consider that

$$
y(x)=\frac{4}{n} \tanh ^{-1}(u(x)),
$$

from which we find

$$
\begin{gathered}
y^{\prime}=\frac{4}{n\left(1-u^{2}\right)} u^{\prime}, \\
y^{\prime \prime}=\frac{4}{n\left(1-u^{2}\right)} u^{\prime \prime}+\frac{8 u}{n\left(1-u^{2}\right)^{2}}\left(u^{\prime}\right)^{2},
\end{gathered}
$$

where prime denotes differentiation with respect to $x$. 
Now, by substituting (3.1) into the sinh term of (1.1), we obtain

$$
y^{\prime \prime}=n \sinh \left(4 \tanh ^{-1}(u(x))\right)=\frac{4 n u\left(u^{2}+1\right)}{(u+1)^{2}(u-1)^{2}} .
$$

Then, equating (3.3) and (3.4), we achieve to the following transformed problem:

$$
\left(1-u^{2}\right) u^{\prime \prime}+2 u\left(u^{\prime}\right)^{2}-n^{2} u\left(1+u^{2}\right)=0
$$

where conditions are obtained by using variable transformation (see (3.1))

$$
u(x)=\tanh \left(\frac{n}{4} y(x)\right)
$$

and substituting original boundary conditions $y(0)=0$ and $y(1)=1$ into above equation, results

$$
u(0)=0, \quad u(1)=\tanh \left(\frac{n}{4}\right)
$$

From (2.4) and (3.5), we can formulate the following homotopy [17-19]:

$$
H(v, p)=(1-p)\left(v^{\prime \prime}-n^{2} v\right)+p\left(\left(1-v^{2}\right) v^{\prime \prime}+2 v\left(v^{\prime}\right)^{2}-n^{2} v\left(1+v^{2}\right)\right)=0
$$

where $p$ is the homotopy parameter.

Substituting (2.5) into (3.8) and equating identical powers of $p$ terms, we obtain

$$
\begin{aligned}
& p^{0}: v_{0}^{\prime \prime}-n^{2} v_{0}=0, \quad v_{0}(0)=0, v_{0}(1)=\tanh \left(\frac{n}{4}\right) \\
& p^{1}: v_{1}^{\prime \prime}-n^{2} v_{1}-n^{2} v_{0}^{3}-v_{0}^{2} v_{0}^{\prime \prime}+2 v_{0}\left(u_{0}^{\prime}\right)^{2}=0, \quad v_{1}(0)=0, v_{1}(1)=0 \\
& p^{2}: v_{2}^{\prime \prime}-n^{2} v_{2}-3 n^{2} v_{0}^{2} v_{1}+4 v_{0} v_{0}^{\prime} v_{1}^{\prime}-2 v_{0} v_{1} v_{0}^{\prime \prime}-v_{0}^{2} v_{1}^{\prime \prime}+2 v_{1}\left(v_{0}^{\prime}\right)^{2}=0, \quad v_{2}(0)=0, v_{2}(1)=0
\end{aligned}
$$


We solve (3.9) by using Maple software, resulting

$$
\begin{aligned}
v_{0}=w & \sinh (n x), \quad w=\frac{\tanh (n / 4)}{\sinh (n)} \\
v_{1}=- & \frac{w^{3} n \exp (-n x)}{2 \exp (2 n)-2}[(x-1)(\exp (2 n(x+1))-1)+(x+1)(\exp (2 n)-\exp (2 n x))], \\
v_{2}=m+ & \frac{q \exp (-3 n x)}{2 n} \\
\times & {\left[\left(\frac{3}{4}+\left(-x^{2}+6 x\right) n^{2}+\left(-\frac{3}{2} x-3\right) n\right) \exp ((4 x+2) n)\right.} \\
& -\frac{1}{8} \exp ((6 x+2) n)+\left(-\frac{3}{4}+\left(6 x+x^{2}\right) n^{2}+\left(-\frac{3}{2} x+3\right) n\right) \exp (2 n(x+1)) \\
& +\left(\frac{3}{4}+\left(-x^{2}+6 x\right) n^{2}+\left(3+\frac{3}{2} x\right) n\right) \exp (2 n x) \\
& +\left(-\frac{3}{4}+\left(6 x+x^{2}\right) n^{2}+\left(\frac{3}{2} x-3\right) n\right) \exp (4 n x) \\
& \left.-\frac{1}{8}+\frac{1}{8} \exp (6 n x)+\frac{1}{8} \exp (2 n)\right]
\end{aligned}
$$

where $m$ is

$$
\begin{aligned}
m=\frac{q(\exp (-n x)-\exp (n x)) \exp (-3 n)}{16 n(\exp (n)-\exp (-n))} \\
\times\left[7 \exp (6 n)+40 n^{2} \exp (6 n)-36 n \exp (6 n)\right. \\
\quad-\exp (8 n)-12 \exp (4 n)+112 n^{2} \exp (4 n)+7 \exp (2 n) \\
\left.\quad+40 n^{2} \exp (2 n)+36 n \exp (2 n)-1\right]
\end{aligned}
$$

and $q$ is

$$
q=-\frac{w^{5} n}{2 \exp (2 n)-2}
$$

Next, calculating the limit when $p \rightarrow 1$, we obtain the second-order approximated solution of (3.5)

$$
u_{2}(x)=\lim _{p \rightarrow 1} v=v_{0}+v_{1}+v_{2}
$$


Finally, from (3.1) and (3.13), the proposed solution of Troesch's problem is

$$
y(x)=\frac{4}{n} \tanh ^{-1}\left(u_{2}(x)\right), \quad 0 \leq x \leq 1, n \geq 0
$$

\subsection{Interval of Solution}

The real branch of $\tanh ^{-1}(z)$ is restricted to the range $-1 \leq z \leq 1$. Therefore, (3.14) requires

$$
-1 \leq u_{2}(x) \leq 1,
$$

where $x$ is delimited by the boundary conditions as

$$
0 \leq x \leq 1
$$

In order to show that (3.14) fullfills the conditions (3.15) and (3.16), we plot (3.13) in Figure 1. From such figure, we can observe that $0<u_{2}(x)<1$ is valid in the intervals of $x \in[0,1]$ and $n \in[0,30]$. Now, from (3.13) and (3.14), we calculate the following limits:

$$
\begin{aligned}
& \lim _{n \rightarrow \infty} u_{2}(1)=1, \\
& \lim _{x \rightarrow 0} u_{2}(x)=0, \\
& \lim _{n \rightarrow 0} y(x)=x .
\end{aligned}
$$

From (3.17), (3.18), (3.19), and Figure 1, we can conclude that the maximum value of (3.13) is 1 in the range of $0 \leq n \leq \infty$. Therefore, (3.13) fullfills (3.15) in the range given by (3.16). Additionally, limit (3.19) shows that for $n=0$, the presented solution (3.14) becomes the exact/trivial solution for (1.1).

\section{Numerical Simulation and Discussion}

In the case of $n=0.5$ (see Table 1), we can observe that the lowest average absolute relative error (A.A.R.E.) is for LDTM [16], followed closely by the proposed solution (3.14). A possible reason can lie in the fact that (3.14) is a second-order approximation, while LDTM is of third order. For $n=1$ (see Table 2), there is a change now the lowest A.A.R.E. is for the proposed solution (3.14), followed by LDTM solution. For both cases, the other approximations ADM [13], HPM [12], HPM [11], and HAM [14] have lower accuracy than (3.14). Equation (3.14) did not require an adjustment parameter, unlike LDTM solution, which required a specific adjustment parameter calculated for each value of Troesch's parameter $n$. Therefore, the proposed solution is easier to use than LDTM solution.

In Table 3, we can observe a comparison of (3.14) with numerical solution [15], and other solutions obtained by HAM [14], VIM [15], and HPM [12] for $n=10$. Approximation (3.14) has the lowest A.A.R.E. from all above solutions, followed by VIM approximation. 


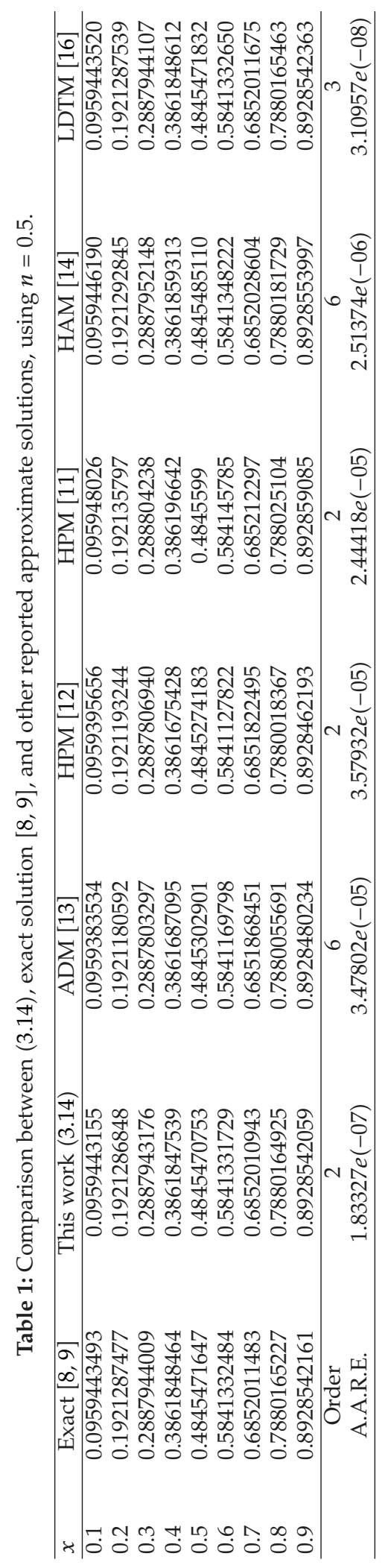




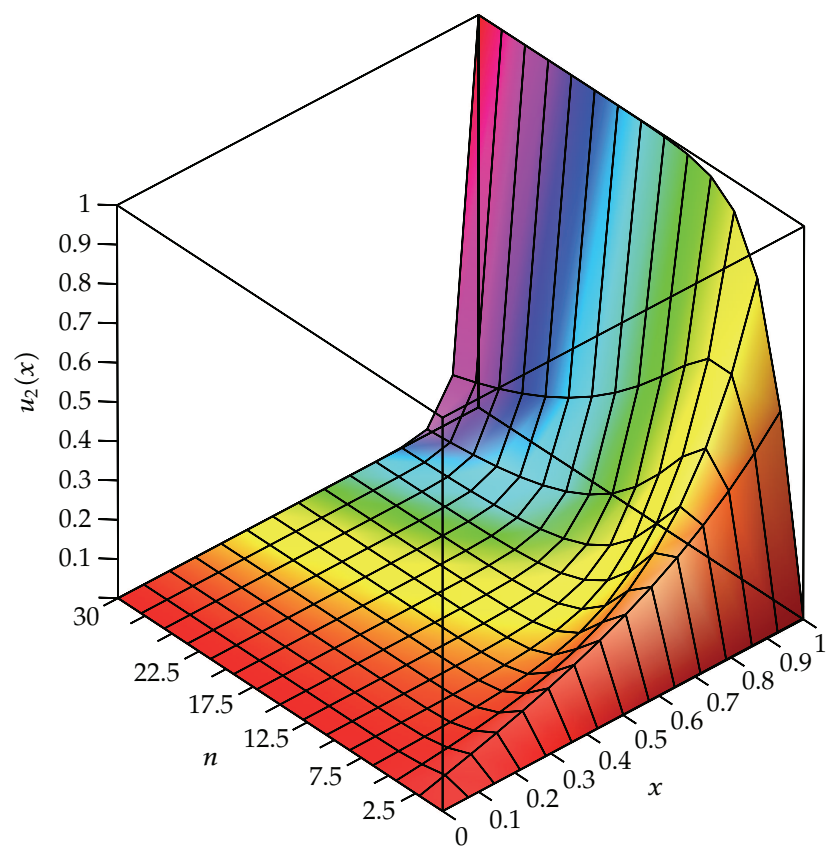

Figure 1: Plot for (3.13) in the range of $x \in[0,1]$ and $n \in[0,30]$.

Table 2: Comparison between (3.14), exact solution [8, 9], and other reported approximate solutions, using $n=1$.

\begin{tabular}{|c|c|c|c|c|c|c|c|}
\hline$x$ & Exact [8] & $\begin{array}{c}\text { This work } \\
(3.14)\end{array}$ & ADM [13] & HPM [12] & HPM [11] & HAM [14] & LDTM [16] \\
\hline 0.1 & 0.0846612565 & 0.08466075858 & 0.084248760 & 0.0843817004 & 0.084934415 & 0.0846732692 & 0.08466308972 \\
\hline 0.2 & 0.1701713582 & 0.1701704581 & 0.169430700 & 0.1696207644 & 0.170697546 & 0.1701954538 & 0.1701750442 \\
\hline 0.3 & 0.2573939080 & 0.2573927827 & 0.256414500 & 0.2565929224 & 0.258133224 & 0.2574302342 & 0.2573994845 \\
\hline 0.4 & 0.3472228551 & 0.3472217324 & 0.346085720 & 0.3462107378 & 0.348116627 & 0.3472715981 & 0.3472303763 \\
\hline 0.5 & 0.4405998351 & 0.4405989511 & 0.439401985 & 0.4394422743 & 0.44157274 & 0.4406610140 & 0.4406093753 \\
\hline 0.6 & 0.5385343980 & 0.5385339413 & 0.537365700 & 0.5373300622 & 0.539498234 & 0.5386072529 & 0.5385460046 \\
\hline 0.7 & 0.6421286091 & 0.6421286573 & 0.641083800 & 0.6410104651 & 0.642987984 & 0.7526899495 & 0.6421421393 \\
\hline 0.8 & 0.7526080939 & 0.7526085475 & 0.751788000 & 0.7517335467 & 0.753267551 & 0.7526899495 & 0.7526226886 \\
\hline \multirow[t]{3}{*}{0.9} & 0.8713625196 & 0.8713630450 & 0.870908700 & 0.8708835371 & 0.871733059 & 0.8714249118 & 0.8713748860 \\
\hline & Order & 2 & 6 & 2 & 2 & 6 & 3 \\
\hline & A.A.R.E. & $2.54568 e(-06)$ & 0.002714577 & 0.002320107 & 0.002044737 & 0.019244326 & $2.05 e(-05)$ \\
\hline
\end{tabular}

Besides, HAM [14] has an relatively poor value of A.A.R.E., despite the fact that it is a sixthorder approximation. Furthermore, HPM [12] shows divergence from the numerical solution. In addition, (3.14) do not require an adjustment parameter, nevertheless, VIM solution required a specific adjustment parameter calculated for each value of Troesch's parameter $n$. Therefore, the proposed solution is easier to use than VIM solution.

In Table 4, is presented a comparison of initial slope $y^{\prime}(0)$ and the results reported in $[4,5,15,28,29]$ for the range of $1 \leq n \leq 20$; resulting that the proposed solution is the only one reported in literature with high accuracy in the complete aforementioned range. Moreover, in 
Table 3: Comparison between (3.14), numerical solution [15], and other reported approximate solutions, using $n=10$.

\begin{tabular}{cccccc}
\hline$x$ & Numerical [15] & This work (3.14) & HAM [14] & VIM [15] & HPM [12] \\
\hline 0.100 & $4.211183679705 e(-05)$ & $4.211189927237 e(-05)$ & $4.19038 e(-05)$ & $4.211189936715 e(-05)$ & 17.617500000 \\
0.200 & $1.299639238293 e(-04)$ & $1.299641158237 e(-04)$ & $1.32094 e(-04)$ & $1.299641161162 e(-04)$ & 33.693333333 \\
0.300 & $3.589778855481 e(-04)$ & $3.589784013896 e(-04)$ & $3.60051 e(-04)$ & $3.589784021976 e(-04)$ & 46.785833333 \\
0.400 & $9.779014227050 e(-04)$ & $9.779027718029 e(-04)$ & $9.24818 e(-04)$ & $9.779027740037 e(-04)$ & 55.653333333 \\
0.500 & $2.659017178062 e(-03)$ & $2.659020490351 e(-03)$ & $2.57642 e(-03)$ & $2.659020496335 e(-03)$ & 59.354166667 \\
0.600 & $7.228924695208 e(-03)$ & $7.228931212877 e(-03)$ & $7.78968 e(-03)$ & $7.228931229141 e(-03)$ & 57.346666667 \\
0.700 & $1.966406025665 e(-02)$ & $1.966406309701 e(-02)$ & $2.22598 e(-02)$ & $1.966406314118 e(-02)$ & 49.589166667 \\
0.800 & $5.373032958567 e(-02)$ & $5.373032935060 e(-02)$ & $5.59715 e(-02)$ & $5.373032947016 e(-02)$ & 36.640000000 \\
0.900 & $1.521140787863 e(-01)$ & $1.521140764047 e(-01)$ & $1.28433 e(-01)$ & $1.521140767248 e(-01)$ & 19.757500000 \\
0.999 & $8.889931171768 e(-01)$ & $8.889931181558 e(-01)$ & - & $8.889931185202 e(-01)$ & - \\
\hline \multicolumn{7}{c}{ Order } & 2 & 6 & 2 & 2 \\
A.A.R.E. & $8.09027 e(-07)$ & 0.057403529 & $8.1021 e(-07)$ & 99824.7587
\end{tabular}

order to compare the derivative of (3.14) for $n>20$, we use the approximate $y^{\prime}(0)$ reported in [29]

$$
y^{\prime}(0)=10^{-12} \exp (29.71-n), \quad y^{\prime}(0)<10^{-4},
$$

resulting in a remarkable accuracy for the range $n \in[22,1 e(+12)]$.

In the same fashion, in Table 5, we compare $y^{\prime}(1)$ for proposed solution and other reported numerical solutions $[4,5,28,30]$. The results shows that (3.14) has a good accuracy for $y^{\prime}(1)$ at least in the range $n \in[1,20]$.

Figure 2 results from Tables 1, 2, and 3 and Maple routines of numerical solution of differential equations. We can observe the overlap of numerical solution and (3.14), for different values of $n \in[0.5,10]$. Therefore, (3.14) is a high accurate approximate solution, valid for a wide range of values of Troesch's parameter.

Further research should be performed in order to verify the accuracy of (3.14) for large values of $n$. Nevertheless, as we know from above discussion $y^{\prime}(0)$ and $y^{\prime}(1)$ are valid and accurate in the ranges $1 \leq n \leq 1 e(+12)$ and $1 \leq n \leq 20$, respectively, therefore, we can expect a high accuracy of (3.14) in the same ranges.

\section{Concluding Remarks}

In this work, we obtained an approximate solution for Troesch's problem. Besides, we presented a comparison between the numerical solution, the proposed solution, and other approximations reported in the literature. The numerical and graphical results show that the proposed solution is the most accurate one, for a wide range of values of Troesch's parameter $(n)$. Moreover, Troesch's problem approximation exhibit a remarkable accuracy for $y^{\prime}(0)$ at least in the range $1 \leq n \leq 1 e(+12)$. In the same fashion, $y^{\prime}(1)$ exhibits a good accuracy at least in the range $1 \leq n \leq 20$. Accordingly, we can expect a good accuracy of proposed solution for the range $0 \leq n \leq 1 e(+12)$ and maybe for even more larger values of $n$. Additionally, the 


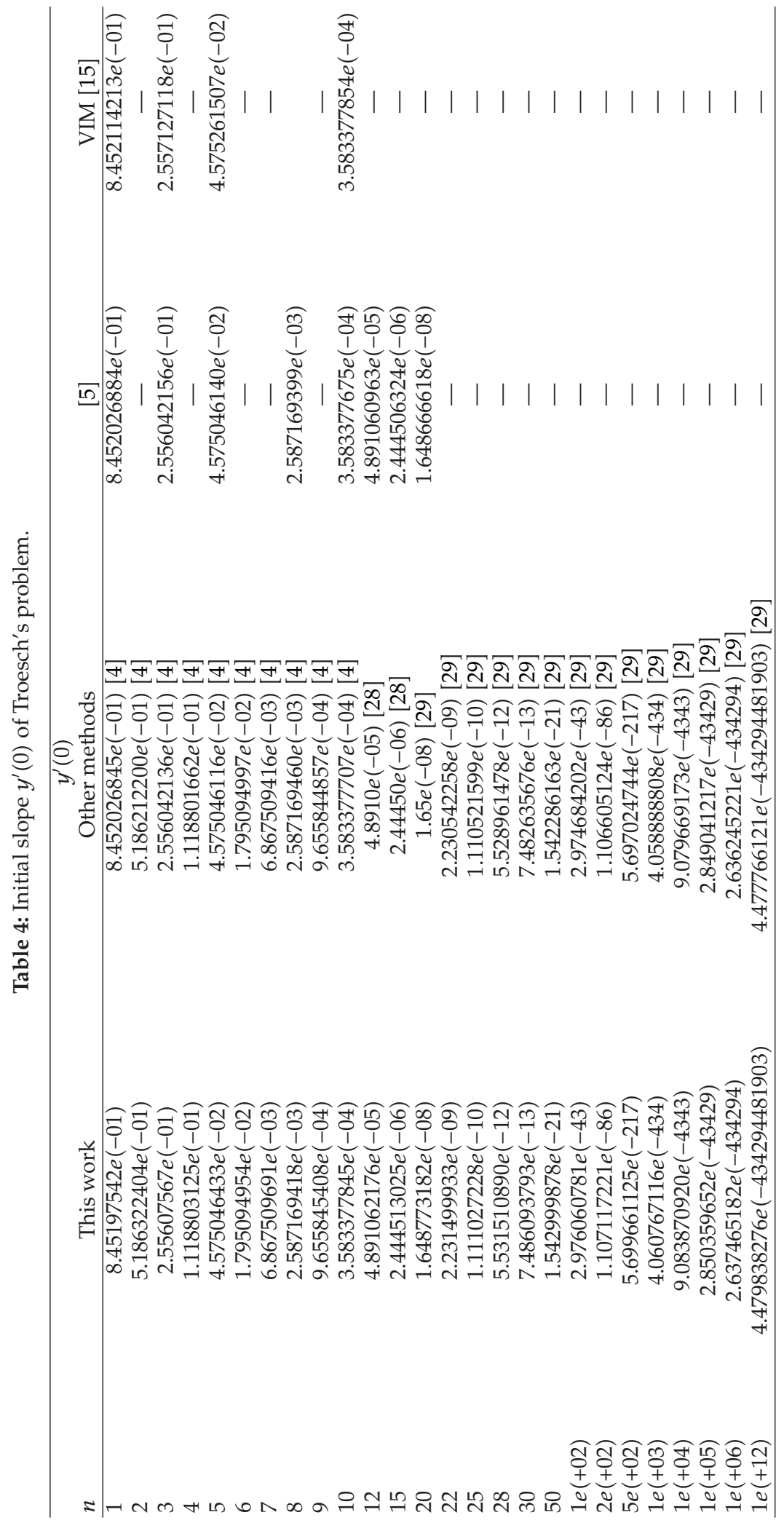


Table 5: Slope $y^{\prime}(1)$ of Troesch's problem.

\begin{tabular}{lccc}
\hline & & $y^{\prime}(1)$ & \\
$n$ & This work & Other methods & {$[5]$} \\
\hline 1 & $1.341828780 e(+00)$ & $1.341837966 e(+00)[4]$ & $1.341837867 e(+00)$ \\
2 & $2.406790318 e(+00)$ & $2.406939711 e(+00)[4]$ & - \\
3 & $4.266151411 e(+00)$ & $4.266223175 e(+00)[4]$ & $4.266222862 e(+00)$ \\
4 & $7.254574096 e(+00)$ & $7.254582910 e(+00)[4]$ & - \\
5 & $1.210049478 e(+01)$ & $1.210049568 e(+01)[4]$ & $1.210049546 e(+01)$ \\
6 & $2.003575791 e(+01)$ & $2.003575367 e(+01)[4]$ & - \\
7 & $3.308525498 e(+01)$ & $3.308526669 e(+01)[4]$ & - \\
8 & $5.457983465 e(+01)$ & $5.461322834 e(+01)[4]$ & $5.457983447 e(+01)$ \\
9 & $9.000602248 e(+01)$ & $9.000618074 e(+01)[4]$ & - \\
10 & $1.484064126 e(+02)$ & $1.484065422 e(+02)[4]$ & $1.484064212 e(+02)$ \\
12 & $4.034263503 e(+02)$ & $4.034263147 e(+02)[28]$ & $4.034263147 e(+02)$ \\
15 & $1.808042673 e(+03)$ & $1.808041861 e(+03)[28]$ & $1.808041861 e(+03)$ \\
20 & $2.202629966 e(+04)$ & $2.20264657 e(+04)[30]$ & $2.202646577 e(+04)$ \\
\hline
\end{tabular}

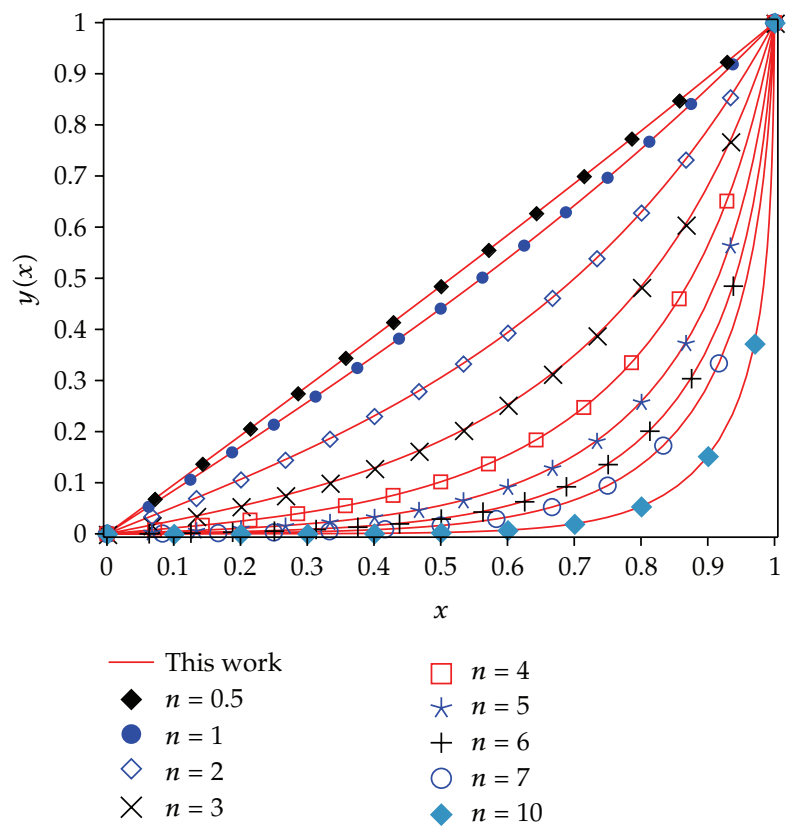

Figure 2: Numerical solution (different symbols) of (1.1) and proposed solution (3.14) (solid line) for different values of $n$.

proposed approximated solution does not require any adjustment parameter as reported for solutions obtained by using LDTM and VIM methods, which makes our proposed solution easier to use than those approximations. Finally, further research is necessary in order to verify the accuracy of our proposed approximation for large values of $n$. 


\section{Acknowledgments}

The authors gratefully acknowledge the financial support of the National Council for Science and Technology of Mexico (CONACYT) through Grant CB-2010-01 no. 157024. The authors would like to thank Rogelio-Alejandro Callejas-Molina and Roberto Ruiz-Gomez for their contribution to this project.

\section{References}

[1] E. S. Weibel, "On the confinement of a plasma by magnetostatic fields," Physics of Fluids, vol. 2, no. 1, pp. 52-56, 1959.

[2] D. Gidaspow and B. S. Baker, "A model for discharge of storage batteries," Journal of the Electrochemical Society, vol. 120, no. 8, pp. 1005-1010, 1973.

[3] V. S. Markin, A. A. Chernenko, Y. A. Chizmadehev, and Y. G. Chirkov, "Aspects of the theory of gas porous electrodes," in Fuel Cells: Their Electrochemical Kinetics, pp. 22-33, Consultants Bureau, New York, NY, USA, 1966.

[4] S. M. Roberts and J. S. Shipman, "On the closed form solution of Troesch's problem," Journal of Computational Physics, vol. 21, no. 3, pp. 291-304, 1976.

[5] S.-H. Chang, "Numerical solution of Troesch's problem by simple shooting method," Applied Mathematics and Computation, vol. 216, no. 11, pp. 3303-3306, 2010.

[6] S. A. Khuri and A. Sayfy, "Troesch's problem: a b-spline collocation approach," Mathematical and Computer Modelling, vol. 54, no. 9-10, pp. 1907-1918, 2011.

[7] M. Zarebnia and M. Sajjadian, "The sinc-galerkin method for solving troesch's problem," Mathematical and Computer Modelling, vol. 56, no. 9-10, pp. 218-228, 2012.

[8] U. Erdogan and T. Ozis, "A smart nonstandard finite difference scheme for second order nonlinear boundary value problems," Journal of Computational Physics, vol. 230, no. 17, pp. 6464-6474, 2011.

[9] Y. Lin, J. A. Enszer, and M. A. Stadtherr, "Enclosing all solutions of two-point boundary value problems for ODEs," Computers and Chemical Engineering, vol. 32, no. 8, pp. 1714-1725, 2008.

[10] D. J. Jones, "Solution of Troesch's, and other, two point boundary value problems by shooting techniques," Journal of Computational Physics, vol. 12, no. 3, pp. 429-434, 1973.

[11] S. H. Mirmoradi, I. Hosseinpour, S. Ghanbarpour, and A. Barari, "Application of an approximate analytical method to nonlinear Troesch's problem," Applied Mathematical Sciences, vol. 3, no. 29-32, pp. 1579-1585, 2009.

[12] X. Feng, L. Mei, and G. He, "An efficient algorithm for solving Troesch's problem," Applied Mathematics and Computation, vol. 189, no. 1, pp. 500-507, 2007.

[13] E. Deeba, S. A. Khuri, and S. Xie, "An algorithm for solving boundary value problems," Journal of Computational Physics, vol. 159, no. 2, pp. 125-138, 2000.

[14] H. N. Hassan and M. A. El-Tawil, "An efficient analytic approach for solving two-point nonlinear boundary value problems by homotopy analysis method," Mathematical Methods in the Applied Sciences, vol. 34, no. 8, pp. 977-989, 2011.

[15] S.-H. Chang, "A variational iteration method for solving Troesch's problem," Journal of Computational and Applied Mathematics, vol. 234, no. 10, pp. 3043-3047, 2010.

[16] S. A. Khuri, "A numerical algorithm for solving Troesch's problem," International Journal of Computer Mathematics, vol. 80, no. 4, pp. 493-498, 2003.

[17] J.-H. He, "Comparison of homotopy perturbation method and homotopy analysis method," Applied Mathematics and Computation, vol. 156, no. 2, pp. 527-539, 2004.

[18] J.-H. He, "An elementary introduction to the homotopy perturbation method," Computers $\mathcal{E}$ Mathematics with Applications, vol. 57, no. 3, pp. 410-412, 2009.

[19] J.-H. He, "Homotopy perturbation technique," Computer Methods in Applied Mechanics and Engineering, vol. 178, no. 3-4, pp. 257-262, 1999.

[20] H. Vázquez-Leal, R. Castaneda-Sheissa, U. Filobello-Nino, A. Sarmiento-Reyes, and J. Sanchez Orea, "High accurate simple approximation of normal distribution integral," Mathematical Problems in Engineering, vol. 2012, Article ID 124029, 22 pages, 2012.

[21] H. Vázquez-Leal, U. Filobello-Niño, R. Castañeda-Sheissa, L. Hernández-Martínez, and A. Sarmiento-Reyes, "Modified HPMs inspired by homotopy continuation methods," Mathematical Problems in Engineering, vol. 2012, Article ID 309123, 19 pages, 2012. 
[22] U. Filobello-Nino, H. Vázquez-Leal, R. Castaneda-Sheissa et al., "An approximate solution of blasius equation by using hpm method," Asian Journal of Mathematics and Statistics, vol. 5, pp. 50-59, 2012.

[23] Y. Khan, Q. Wu, N. Faraz, and A. Yildirim, "The effects of variable viscosity and thermal conductivity on a thin film flow over a shrinking/stretching sheet," Computers E Mathematics with Applications, vol. 61, no. 11, pp. 3391-3399, 2011.

[24] N. Faraz and Y. Khan, "Analytical solution of electrically conducted rotating flow of a second grade fluid over a shrinking surface," Ain Shams Engineering Journal, vol. 2, no. 3-4, pp. 221-226, 2011.

[25] Y. Khan, Q. Wu, N. Faraz, A. Yildirim, and M. Madani, "A new fractional analytical approach via a modified Riemann-Liouville derivative," Applied Mathematics Letters, vol. 25, no. 10, pp. 1340-1346, 2012.

[26] Y. Khan, H. Vázquez-Leal, and Q. Wu, "An efficient iterated method for mathematical biology model," Neural Computing and Applications. In press.

[27] U. Filobello-Nino, H. Vazquez-Leal, Y. Khan et al., "HPM applied to solve nonlinear circuits: a study case," Applied Mathematical Sciences, vol. 6, no. 85-88, pp. 4331-4344, 2012.

[28] B. A. Troesch, "A simple approach to a sensitive two-point boundary value problem," Journal of Computational Physics, vol. 21, no. 3, pp. 279-290, 1976.

[29] M. Kubiček and V. Hlaváček, "Solution of Troesch's two-point boundary value problem by shooting technique," Journal of Computational Physics, vol. 17, no. 1, pp. 95-101, 1975.

[30] J. A. Snyman, "Continuous and discontinuous numerical solutions to the Troesch problem," Journal of Computational and Applied Mathematics, vol. 5, no. 3, pp. 171-175, 1979.

[31] H. Koçak, A. Yıldırım, D. H. Zhang, and S. T. Mohyud-Din, “The comparative Boubaker polynomials expansion scheme (BPES) and homotopy perturbation method (HPM) for solving a standard nonlinear second-order boundary value problem," Mathematical and Computer Modelling, vol. 54, no. 1-2, pp. 417-422, 2011.

[32] A. Yildirim and H. Koçak, "Homotopy perturbation method for solving the space-time fractional advection-dispersion equation," Advances in Water Resources, vol. 32, no. 12, pp. 1711-1716, 2009.

[33] A. Yildirim and H. Koçak, "Series solution of the Smoluchowski's coagulation equation," Journal of King Saud University. Science, vol. 23, no. 23, pp. 183-189, 2010.

[34] H. Koçak and A. Yildirim, "Numerical solution of 3D Green's function for the dynamic system of anisotropic elasticity," Physics Letters, Section A, vol. 373, no. 35, pp. 3145-3150, 2009.

[35] J. Biazar and H. Aminikhah, "Study of convergence of homotopy perturbation method for systems of partial differential equations," Computers E Mathematics with Applications, vol. 58, no. 11-12, pp. 2221-2230, 2009.

[36] J. Biazar and H. Ghazvini, "Convergence of the homotopy perturbation method for partial differential equations," Nonlinear Analysis. Real World Applications, vol. 10, no. 5, pp. 2633-2640, 2009.

[37] J. Biazar, F. Badpeima, and F. Azimi, "Application of the homotopy perturbation method to ZakharovKuznetsov equations," Computers E Mathematics with Applications, vol. 58, no. 11-12, pp. 2391-2394, 2009.

[38] J. Biazar and H. Ghazvini, “Exact solutions for non-linear Schrödinger equations by He's homotopy perturbation method," Physics Letters. A, vol. 366, no. 1-2, pp. 79-84, 2007.

[39] J. Biazar, H. Ghazvini, and M. Eslami, "He's homotopy perturbation method for systems of integrodifferential equations," Chaos, Solitons and Fractals, vol. 39, no. 3, pp. 1253-1258, 2009.

[40] J. Biazar, M. Eslami, and H. Aminikhah, "Application of homotopy perturbation method for systems of Volterra integral equations of the first kind," Chaos, Solitons and Fractals, vol. 42, no. 5, pp. 3020 3026, 2009.

[41] J. Biazar and H. Ghazvini, "He's homotopy perturbation method for solving systems of Volterra integral equations of the second kind," Chaos, Solitons and Fractals, vol. 39, no. 2, pp. 770-777, 2009.

[42] J. Biazar and H. Ghazvini, "Numerical solution for special non-linear Fredholm integral equation by HPM," Applied Mathematics and Computation, vol. 195, no. 2, pp. 681-687, 2008.

[43] M. Madani, M. Fathizadeh, Y. Khan, and A. Yildirim, "On the coupling of the homotopy perturbation method and Laplace transformation," Mathematical and Computer Modelling, vol. 53, no. 9-10, pp. 19371945, 2011.

[44] N. Faraz, Y. Khan, and A. Yildirim, "Analytical approach to two-dimensional viscous flow with a shrinking sheet via variational iteration algorithm-II," Journal of King Saud University. Science, vol. 23, no. 1, pp. 77-81, 2011.

[45] D. D. Ganji and A. Rajabi, "Assessment of homotopy-perturbation and perturbation methods in heat radiation equations," International Communications in Heat and Mass Transfer, vol. 33, no. 3, pp. 391-400, 2006. 
[46] M. Rafei, D. D. Ganji, and H. Daniali, "Solution of the epidemic model by homotopy perturbation method," Applied Mathematics and Computation, vol. 187, no. 2, pp. 1056-1062, 2007.

[47] D. D. Ganji, "The application of He's homotopy perturbation method to nonlinear equations arising in heat transfer," Physics Letters. A, vol. 355, no. 4-5, pp. 337-341, 2006.

[48] D. D. Ganji and A. Sadighi, "Application of homotopy-perturbation and variational iteration methods to nonlinear heat transfer and porous media equations," Journal of Computational and Applied Mathematics, vol. 207, no. 1, pp. 24-34, 2007.

[49] M. Sheikholeslami, H. R. Ashorynejad, D. D. Ganji, and A. Yildirim, "Homotopy perturbation method for three-dimensional problem of condensation film on inclined rotating disk," Scientia Iranica, vol. 19, no. 3, pp. 437-442, 2012.

[50] D. D. Ganji, H. Tari, and M. B. Jooybari, "Variational iteration method and homotopy perturbation method for nonlinear evolution equations," Computers $\mathcal{E}$ Mathematics with Applications, vol. 54, no. 7-8, pp. 1018-1027, 2007.

[51] Y. Khan, H. Vazquez-Leal, and N. Faraz, "An auxiliary parameter method using adomian polynomials and laplace transformation for nonlinear differential equations," Applied Mathematical Modelling. In press.

[52] H. Vazquez-Leal, "Rational homotopy perturbation method," Journal of Applied Mathematics, vol. 2012, Article ID 490342, 14 pages, 2012.

[53] Y. Khan, H. Vazquez-Leal, and L. Hernandez-Martinez, "Removal of noise oscillation term appearing in the nonlinear equation solution," Journal of Applied Mathematics, vol. 2012, Article ID 387365, 9 pages, 2012. 


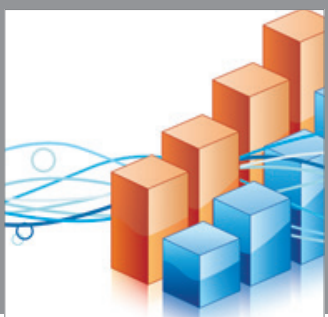

Advances in

Operations Research

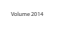

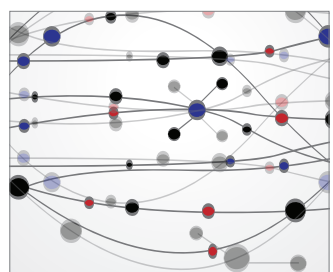

\section{The Scientific} World Journal
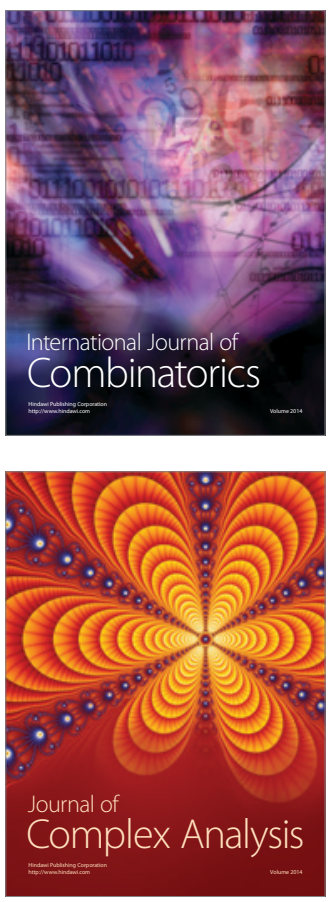

International Journal of

Mathematics and

Mathematical

Sciences
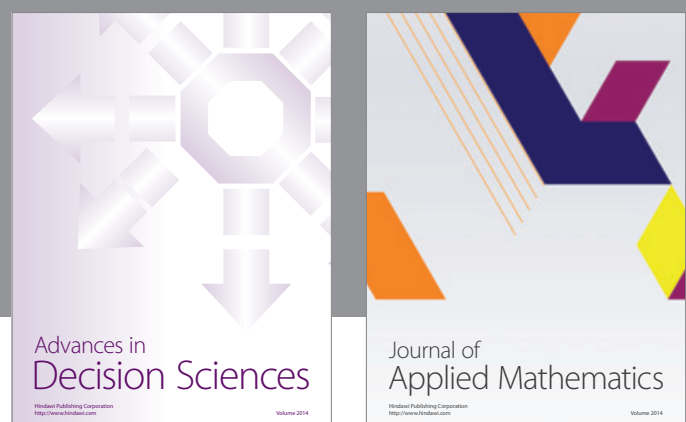

Journal of

Applied Mathematics
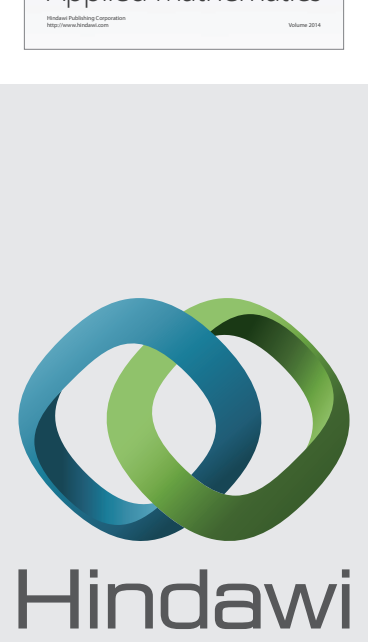

Submit your manuscripts at http://www.hindawi.com
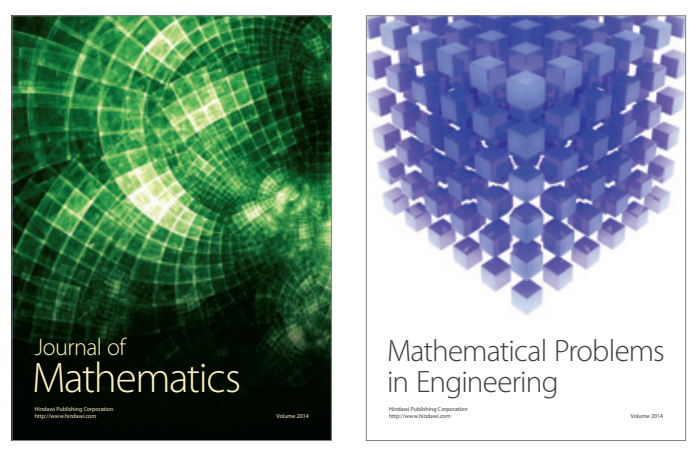

Mathematical Problems in Engineering
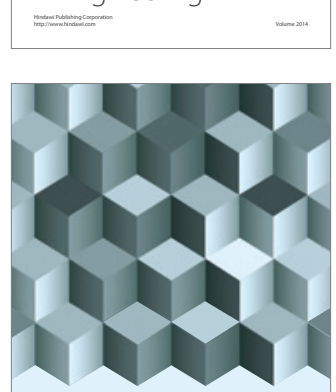

Journal of

Function Spaces
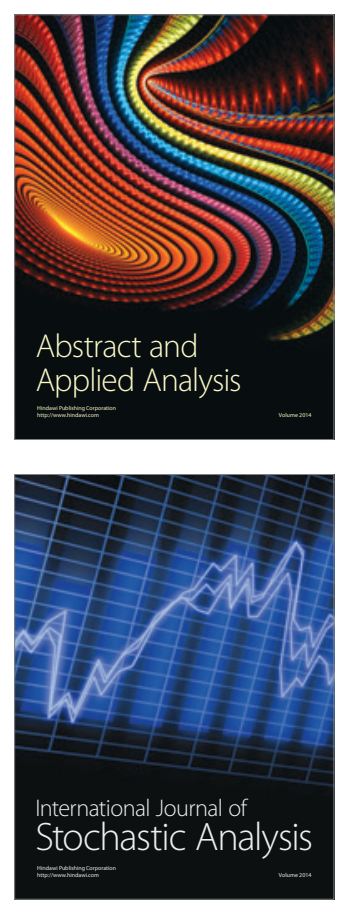

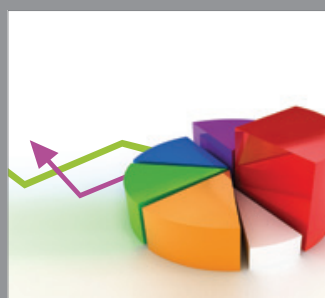

ournal of

Probability and Statistics

Promensencen
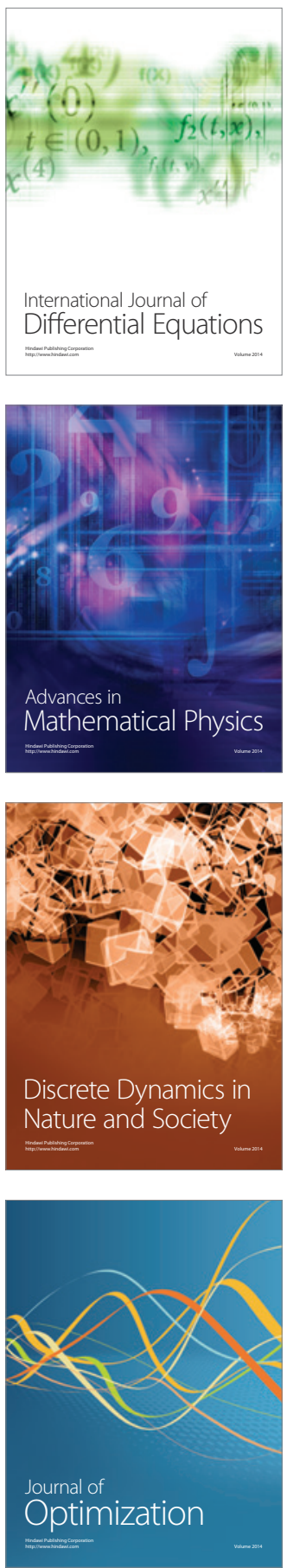